\title{
Spontaneous-braking and lane-changing effect on traffic congestion using cellular automata model applied to the two-lane traffic
}

\author{
Kohei Arai ${ }^{1}$ \\ Graduate School of Science and Engineering \\ Saga University \\ Saga City, Japan
}

\author{
Steven Ray Sentinuwo ${ }^{2}$ \\ Graduate School of Science and Engineering \\ Saga University \\ Saga City, Japan
}

\begin{abstract}
In the real traffic situations, vehicle would make a braking as the response to avoid collision with another vehicle or avoid some obstacle like potholes, snow, or pedestrian that crosses the road unexpectedly. However, in some cases the spontaneous-braking may occur even though there are no obstacles in front of the vehicle. In some country, the reckless driving behaviors such as sudden-stop by public-buses, motorcycle which changing lane too quickly, or tailgating make the probability of braking getting increase. The new aspect of this paper is the simulation of braking behavior of the driver and presents the new Cellular Automata model for describing this characteristic. Moreover, this paper also examines the impact of lane-changing maneuvers to reduce the number of traffic congestion that caused by spontaneous-braking behavior of the vehicles.
\end{abstract}

Keywords- spontaneous-braking; traffic congestion; cellular automata; two-lane trafficcomponent.

\section{INTRODUCTION}

The study of traffic flow has received a lot of attention for the past couple of decades. The simulations of traffic congestion become the most important aspect in the field of traffic analysis and modeling. Traffic congestion can be defined as the saturation condition of road network that occurs as increased traffic volume or interruption on the road, and is characterized by slower speed, longer trip times, and increased vehicular queuing. The investigated situations in the real traffic condition are those of traffic congestion caused by some main reason, such as insufficient road capacity, incidents, work zones (e.g., road maintenance or constructions near the road that requires space), weather events (e.g., in the case of rain or snow) which can hampers visibility therefore a driver have to slowdown its vehicle to compensate, or emergencies situations (e.g., hurricanes or severe snowstorms). However, in this paper, we concern to investigate the effect of individual braking behavior of the driver towards traffic congestion.

In more detail, this paper interests to describe and reproduce the characteristic of spontaneous-braking probability and its effects to the traffic behavior. In the real traffic situations, vehicle would make a braking as the response to avoid collision with another vehicle or avoid some obstacle like potholes, snow, or pedestrian that crosses the road unexpectedly. However, in some cases the spontaneousbraking may occur even if there are no obstacles in front of the vehicle. In some country, the reckless driving behaviors such as sudden-stop by public-buses, motorcycle which changing lane too quickly, or tailgating make the probability of braking getting increase.

One of the famous microscopic models for the simulation of road traffic flow is Cellular Automata (CA) model. In comparison with another microscopic model, the CA model proposes an efficient and fast performance when used in computer simulation [18]. CA is a dynamic model developed to model and simulates complex dynamical system. The set of CA rules may illustrate complex evolution patterns, such as time and space evolution in a system. Those evolutions can be shown just by use simple rules of CA. Furthermore, the utilization of CA successfully explains the phenomenon of transportation. These so-called traffic cellular automata (TCA) are dynamical systems that are discrete in nature and powerful to capture all previously mentioned basic phenomena that occur in traffic flows [18]. The one dimensional cellular automata model for single lane freeway traffic introduced by Nagel and Schreckenberg (NaSch) [1] is simple and elegant that captures the transition from laminar flow to start-stop waves with increasing vehicle density. The space of CA is discrete and consists of a regular grid of cells, each one of which can be in one of finite number of possible states. The number and array of cells in the grid depends on the specific transportation behavior that is described. The simplicity of the NaSch model has prompted the use of it for studying many traffic situations.

This paper presents a new Cellular Automata model for describing the phenomena of spontaneous-braking behavior and lane-changing character in traffic flow. In this model, we investigate the effect of spontaneous-braking probability and lane-changing maneuver in two-lane highway with one-way traffic character. This proposed model extends the NaSch model that first introduced CA for traffic simulation. The set of rules in NaSch model are modified to better capture and describe the behavior of the driver while making spontaneousbraking and lane-changing maneuver in traffic flow. The base deceleration rule of $\mathrm{NaSch}$ model is applicable only to stationary vehicles, which is vehicles that are blocked by the 
leading vehicle in the previous time step. This rule is not applicable to two conditions, in the condition of those vehicles which are stopped due to spontaneous-braking behavior, and in the two-lane highway that allows vehicle to make lanechanging maneuvers. Compared with the original NaSch model, this proposed model exhibits spontaneous-braking probabilities effect combined with acceleration, deceleration, and lane-changing maneuvers effects. Though it is well known that spontaneous-braking is extremely reducing the local speed of vehicles, the impact on the global system has not been studied.

This paper uses a two-lane highway character with a periodic boundary condition. The periodic boundary approach has been used to conserve the number of vehicles and the stability of the model. The goal of this paper is to analyze the phenomena of spontaneous-braking behavior in traffic flow then propose a new cellular automata model to describing this phenomena. Moreover, this paper also investigates the impact of lane-changing maneuvers towards traffic congestion that is caused by spontaneous-braking behavior.

This paper is organized as follows. Some studies relating with CA based traffic flow is quick reviewed in Section 2. Section 3 presents a short description of the theoretical aspect of traffic CA model. Section 4 explains about the proposed model. Section 5 contains simulation process and the results in the form of fundamental diagrams and space-time diagrams. Finally, Section 6 contains conclusion and a summary of findings.

\section{RELATED RESEARCH WORKS}

The one dimensional cellular automata model for single lane freeway traffic introduced by Nagel and Schreckenberg (NaSch) [1] is a probabilistic CA model that captures the transition from laminar flow to start-stop waves with increasing vehicle density. NaSch model update the state of cells synchronously in discrete time steps. There is a finite set of local interaction rules. This set of rules manages the new state of a cell by taking into account the actual state of the cell and its neighbor cells. This local interaction allows capture micro-level dynamics and propagates it to macro-level behavior. This single-lane system consists of a onedimensional grid of L sites with periodic boundary conditions. A site can either be occupied, or empty by one vehicle with integer velocity between zero and $v_{\max }$. The velocity of each vehicle is equivalent to the number of sites that a vehicle advances in one update, if there is no obstacle ahead. Each of vehicles moves only in one direction. Refer to the Ricket et. al [6], they outlined the rules of single-lane model. The index $i$ denotes the number of vehicle, $x(i)$ is the position of vehicle $i$, $v(i)$ is the vehicle's current velocity, $v_{d}(i)$ is the maximum speed, $\operatorname{pred}(i)$ is the number of preceding vehicle, $\operatorname{gap}(i)=$ $x(\operatorname{pred}(i))-x(i)-1$ indicates the width of the gap to the predecessor. The rules are applied to all vehicles at the beginning of each time step by simultaneously, which mean using parallel update. Then the vehicles are advanced according to their new velocities [6].

The parallel update rules are the following:

- $v(i) \neq v_{d}(i) \Rightarrow v(i):=v(i)+1$
- $v(i)>\operatorname{gap}(i) \Rightarrow v(i):=\operatorname{gap}(i)$

- $v(i)>0 \Rightarrow$ rand $<p_{d}(i) \Rightarrow v(i):=v(i)-1$

The first rule represents the linear acceleration of each vehicle which is not at the maximum speed to accelerate its speed by one site (cell) until the vehicle has reached its maximum velocity $v_{d}$. Second rule ensures that vehicles having predecessors in their way slowdown in order not to run into them. In this rule, all vehicles are checked for their distance between the vehicle and its predecessor. If the distance is smaller than its speed then the speed is reduced to the number of empty cells between them to avoid the collision. Third rule consider the stochastic noise parameter.

The probability $p_{\mathrm{d}}$ is the probability number of each car to reduce its speed by one unit (cell) per time step. This NaSch model encouraged another study toward traffic flow conditions [2]-[7]. Ricket, et al. [8] investigated a simple model for twolane traffic. Their model introduced the lane changing behavior for two lanes traffic. It was found that the fundamental diagram for each lane is asymmetric but the maximum is shifted towards large values of vehicular density $\rho\left(\rho_{\max }>1 / 2\right)$. They proposed a symmetric rule set where the vehicle changes lanes if the following criteria are fulfilled:

- $v_{\text {move }}>$ gap $_{\text {same }} \rightarrow v_{\text {move }}=\min \left(v_{\mathrm{n}}+1, v_{\max }\right)$

- gap $_{\text {target }}>$ gap $_{\text {same }}$

- gap $_{\text {back }} \geq v_{\text {max }}$

The variable gap $_{\text {same, }}$ gap $_{\text {target, }}$ and gap back $_{\text {denote the }}$ number of unoccupied cells between the vehicle and its predecessor on its current lane, and between the same vehicle and its two neighbor vehicles on the desired lane, respectively.

The advance analysis about lane-changing behavior has been done, which includes symmetric and asymmetric rules of lane-changing [9-14]. Symmetric rule can be considered as rules that threat both lanes equally, while asymmetric rule can be applied in special characters highway, like German highways simulation [15], where lane changes are dominated by right lane rather than left lane. Another studies focus on the effect of lane-changing behavior on a two-lane road in presence of slow vehicle and fast vehicle [13], [16-18]. While the NaSch model could reproduce some of basic phenomenon observed in real traffic situations such as the start-stop waves in congested traffic, but it has been observed that the base NaSch model lacks the ability to produce other more realistic traffic patterns [19].

In this paper, we consider two parameters in traffic behavior; those are the spontaneous-braking behavior and lane-changing maneuver that occurs in the real traffic situation. This proposed model using two-lanes traffic and also adopts the symmetric lane-changing rules.

\section{TrafFic Cellar Automata Model}

Cellular automaton (CA), at the basis of the model presented in this paper, is a discrete model studied in computability theory, mathematics, physics, complexity science, theoretical biology and microstructure modeling. 
Currently, various fields have been using CA models to model the phenomena of their system, such as vehicular traffic flow, pedestrian behavior, escape and panic dynamic, collective behavior, and self-organization. CA model uses a simple approach for modeling and simulation of complex dynamical systems. The behavior of complex systems can be described by considering at the local interactions between their elementary parts. CA decomposes a complex phenomenon into a finite number of elementary processes.

The CA model consists of two components, a cellular space and a set of state. The state of a cell is completely determined by its nearest neighborhood cells. All neighborhood cells have the same size in the lattice. Each cell can either be empty, or is occupied by exactly one node. There is a set of local transition rule that is applied to each cell from one discrete time step to another (i.e., iteration of the system). This parallel updating from local simple interaction leads to the emergence of global complex behavior.

The Nagel-Schreckenberg (NaSch) model is one of the theoretical CA models for the simulation of freeway traffic [1]. This NaSch model known as the simple CA model for illustrate road traffic flow that can reproduce traffic congestion, like slow down car behavior in a high-density road condition. This model shows how traffic congestion can be thought of as an emergent or collective phenomenon due to interactions between cars on the road, when the density of cars is high and so cars are close to each on average. The NaSch model also known as stochastic traffic cellular automaton (STCA) because it included a stochastic term in one of its rules. Like in deterministic traffic CA models (e.g., CA-184 or DFI-TCA), this NaSch model contains a rule that reflect vehicle increasing speed and braking to avoid collision. However, the stochasticity term also introduced in the system by its additional rule. In one of its rules, at each time-step $t$, a random number $\xi(t) \in[0,1]$ is generated from a uniform distribution. This random number is then compared with a stochastic noise parameter $p \in[0,1]$. For it is based on this probability $p$ then a vehicle will slow down to $v(\mathrm{i})-1$ cells/time-step. According to Nagel and Schreckenberg, the randomization rule captures natural speed fluctuations due to human behavior or varying external conditions [20].

\section{PROPOSED METHOD}

This paper extends a probabilistic CA model that introduced by Nagel-Schrekenberg [1] for the description of single-lane highway traffic. While the original NaSch model uses a single lane that is represented by a one-dimensional array of L sites (cells), this paper considers two-lane highway with unidirectional traffic character in periodic boundaries condition. The two-lane model is needed to describe the more realistic traffic condition which has several types of vehicles with multiple desired velocities. In single-lane model, the vehicles with multiple desired velocities just resulting in the platooning effect with slow vehicle being followed by faster ones and the average velocity reduced to the free-flow velocity of the slowest vehicle [8].

The simulation model in this paper presents two additional elements. The first additional element is spontaneous-braking parameter. This element is needed to illustrate the probability of spontaneous-braking behavior of the vehicle that occur in the real traffic situation. The concept of spontaneous-braking probability is introduced for the description of the spontaneous reaction of the drivers while making a spontaneous-braking behavior. This reaction can be caused by several things e.g., as the response to avoid collision with another vehicles, the reckless driving behaviors such as sudden-stop by publicbuses, motorcycle which changing lane too quickly, or tailgating. Those behaviors make the probability of braking getting increase.

In original NaSch model [1], there is no rule accommodate the spontaneous-braking behavior. NaSch model introduced a stochastic noise parameter $p \in[0,1]$ that can make a slowdown vehicle to $v(\mathrm{i})-1$ cells/time-step. However, in real traffic situations this rule is difficult to describe the nature of the braking, especially on spontaneous-braking behavior of the vehicle. In our opinion, the value of braking is a variable number and the spontaneous-braking represent the extreme value of a braking behavior. Thus, the slow-down rule of vehicle $v(\mathrm{i})-1$ cells/time-step cannot describe the characteristic of spontaneous-braking. This paper introduces a new additional rule to represent the behavior of spontaneousbraking by using a spontaneous-braking probability $P_{b}: v(i) \rightarrow$ $v(i)-b_{x}$. Here $b x$ denotes the characteristic of driver while make a braking. The value of $b_{x}$ is equal or less than the current speed $v(i)$. This rule takes into account the dynamic characteristic of the driver while make a braking of its car. Already mentioned before, a two-lane unidirectional highway model with periodic boundary system is used in this computational model. Refer to the discrete NaSch model, a one-dimensional chain of $\mathrm{L}$ cells of length $7.5 \mathrm{~m}$ represents each lane. There are just two possibility states of each cell. Each cell can only be empty or containing by just one vehicle. The speed of each vehicle is integer value between $v=0,1, \ldots$ . $v_{\max }$. In this model, all vehicles are considered as homogeneous then have the same maximum speed $v_{\max }$. In order to investigate the effect of spontaneous-braking behavior then the state of a road cell at the next time-step, from $t$ to $t+$ 1 is dependent on the states of the direct frontal neighborhood cell of the vehicle and the core cell itself of the vehicle. The state of the road cells can be obtained by applying the following rules to all cells (vehicles) by parallel updated:

Acceleration: $v(i) \rightarrow \min \left(v(i)+1, v_{\max }\right)$

Deceleration: $v(i) \rightarrow \min (v(i), \operatorname{gap}(i))$

Spontaneous braking probability $\mathrm{p}_{\mathrm{b}}: v(i) \rightarrow v(i)-b_{x}$ Driving: $x(i) \rightarrow x(i)+v(i)$

As this simulation model try to investigate the effect of spontaneous-braking behavior on traffic flow then this model deliberately eliminates the randomization rule of original NaSch $(v(\mathrm{i})-1$ cells/time-step). Here for the reason to avoid the speed reduction of vehicles caused by this rule that could influence our simulation results. The variable gap $(i)$ indicates the distance between a vehicle $x(i)$ and its predecessor

$x((i)+1) . v \max$ represents the maximum speed of the vehicle.

The second additional element is lane-changing parameter. By using two-lane highway model and applying multiple 
desired velocity types, then this paper also accommodates the lane-changing maneuvers of vehicles. In the real traffic situation, driver tends to make a lane-changing maneuver while encounter traffic congestion along its lane. This paper also intends to evaluate the impact of lane-changing maneuvers towards the traffic congestion that caused by spontaneous-braking behavior of the driver. In this model, the lane-changing maneuver is analogous as the movement of liquid. There is a different from the lane-changing model of Ricket et al. In this model, a vehicle would consider changing its lane only if the vehicles "see" another vehicle on its cell ahead and do so if possible. It means, as long as there is a cell free ahead on their lane then the vehicles would still remain on their lane. This lane-changing model will preserve the deceleration rule in our model that is showed in equation (5).

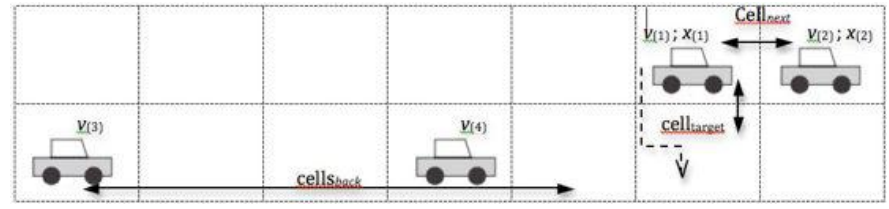

Figure 1. Schematic diagram of a lane-changing operation

The lane-changing rule is applied to vehicles to change from right lane to left lane and conversely. Vehicles are only move sideways and they do not advance. Fig. 1 shows the schematic diagram of lane-changing operation. A vehicle changes to the next lane if all of the following conditions are fulfilled:

- Cell $_{\text {next }}>0$
- Cell $_{\text {target }}=0$
- $x_{(\text {cellsback })}+v_{(\text {cellsback })}{ }^{t+1} \neq$ cell $_{\text {arget }}$

Cell $l_{\text {next }}, \mathrm{Cell}_{\text {target }}$, and $\mathrm{Cell}_{\text {back }}$ are the parameters that inform the state of one cell ahead, state of next cell, and state of cells behind on the other lane, respectively. If one cell is unoccupied or free-cell then its state is 0 . In the real traffic situation, a driver also has to look back on the other lane and estimate the velocity of another cars-behind to avoid a collision. Equation (10) accommodates the driver behavior to estimate the velocity of vehicles before change the lane.

\section{SIMULATIONA DN RESUlTS}

The simulation starts with an initial configuration of $\mathrm{N}$ vehicles, with random distributions of positions on both lanes. This simulation use the same initial velocity for all vehicle $v_{\min }$ $=0$ and the maximum vehicle speed has been set to $v_{\max }=5$ cell/time-step. Many simulations performed with different density $\rho$. The density $\rho$ can be defined as number of cars $\mathrm{N}$ along the highway over number of cells on the highway $\mathrm{L}$. During one simulation, the total number of cars on the highway cannot change. Vehicles go from left to right. If a vehicle arrives on the right boundary then it moves to the left boundary. Fig. 2 illustrates an environment, which exhibits a certain configuration.

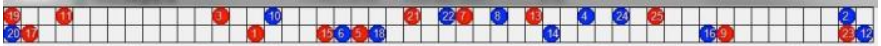

Figure 2. An environment with a certain configuration
This paper divides the analysis into two stages. The first stage investigates the effect of spontaneous-braking on the traffic flow. In this simulation stage, we analyze the traffic flow for the spontaneous-braking probability $b_{p}=0 ; 0.3$; and 0.7. The simulation was running 1000 time steps to let the system reaches its stable condition. The system automatically increase the vehicles density from minimum density $\rho=0$ until maximum density $\rho=100$ percent. Once the transient dies out, then the data extraction was started. The data was analyzed using fundamental diagrams, which plot the velocity of vehicle $v s$ vehicle flow $v s$ global density.

To show the system dynamics then the graph had written the last ten steps for each density before the end of simulation. Fig. 3 and Fig. 4 present the fundamental diagrams of this model. Fig. 3 shows the measurement of the average velocity $v$ $(t)$ over all vehicles at each density. The red color, black color, and blue color of scatter graph present the average velocity in the condition with spontaneous-braking probability $\mathrm{P}_{\mathrm{b}}=0, \mathrm{P}_{\mathrm{b}}=0.3$, and $\mathrm{P}_{\mathrm{b}}=0.7$, respectively.

One can be observed that in the traffic without spontaneous braking probability, the maximum velocity 5 unit of distance per unit of time could be achieved in the density $\rho \leq 0.12$. When the probability of spontaneous-braking increased then the critical density point that maximum velocity can be achieved became lower than normal condition.

For the spontaneous-braking probability $\mathrm{P}_{\mathrm{b}}=0.3$, the critical point of maximum velocity $v_{\max }=5$ is around $\rho=0.04$. While in the situation that spontaneous-braking probability $\mathrm{P}_{\mathrm{b}}$ $=0.7$, the vehicles were very difficult to reach their maximum speed $v_{\max }=5$.

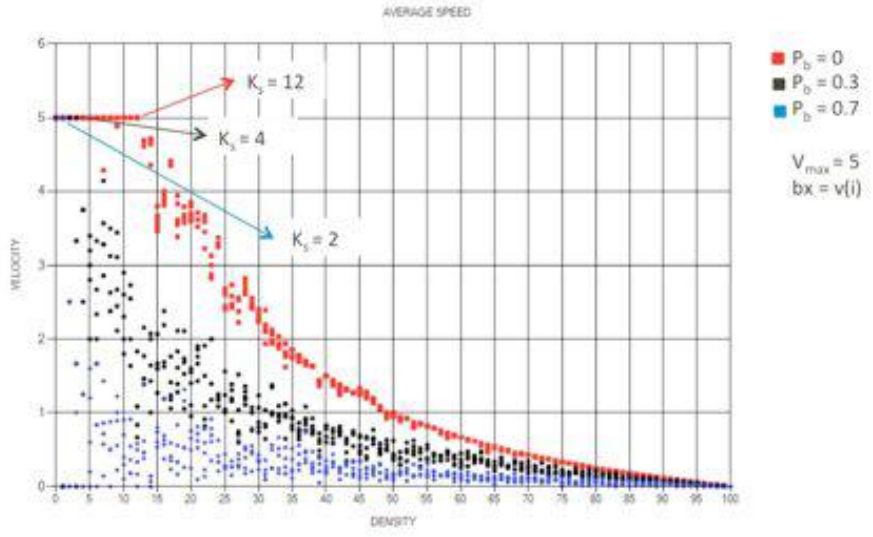

Figure 3. Average velocity (cell/time-step) $v s$ density (cars/highway site)

In the phase after the critical density point of maximum velocity was reached, the vehicles reduced their velocity to synchronize with the gap between them and the vehicle ahead.

However, in the transition phase after the critical density point of maximum velocity, the vehicles still maintained their velocity. Regarding this average velocity graph, the traffic jam obviously appeared when the average velocity $v<1$ cell/time. 


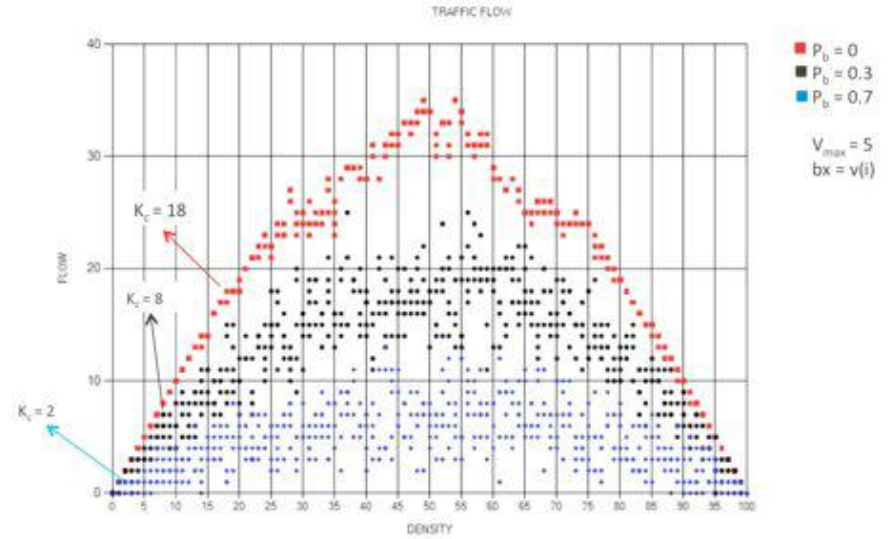

Figure 4. Traffic flow (cars/time step) $v s$ density (cars/highway site)

Fig. 4 illustrates the traffic flow over vehicles density for the spontaneous-braking probability $\mathrm{P}_{\mathrm{b}}=0, \mathrm{P}_{\mathrm{b}}=0.3$, and $\mathrm{P}_{\mathrm{b}}=$ 0.7 , respectively. The traffic flow indicates the number of moving vehicles per unit of time. While the density parameter means the number of vehicles per unit area of the highway. As can be seen from the graph, there is a reduction in traffic flow in the presence of spontaneous-braking parameter. We also consider the critical density $\mathrm{k}_{\mathrm{c}}$ that appeared in each traffic flow. Here, the critical density means a maximum density achievable under free flow. In the traffic flow with $\mathrm{P}_{\mathrm{b}}=0$, the critical density $\mathrm{k}_{\mathrm{c}}$ situated at the density $\rho=0.18$.

The critical density $\mathrm{k}_{\mathrm{c}}$ was getting lower when the spontaneous-braking parameter increased. Below the critical density $\mathrm{k}_{\mathrm{c}}$, all vehicles can make a movement. However, in the density after the critical density point, not every vehicle can move at each time step. This critical density point also indicates when the traffic congestion started to happen. To get an intuitive feel for the dynamics, we provide a set of spacetime diagrams in Fig. 5, Fig. 6, and Fig. 7 for various density values.

The horizontal axis represents space and vertical axis represents the time. In order to get data to analyze, we simulate this model for density $\rho=0.25 ; 0.50$; and 0.75 that represent light traffic, moderate traffic, and heavy traffic situations.

For density $\rho=0.25$, it can be seen that the spontaneousbraking behavior has given a significant impact to produce traffic congestion (Fig. 5). The single vertical line which is shown in these time-space diagrams represents a stationary vehicle that is making a spontaneous-braking behavior. In the traffic with density value $\rho=0.50$, there is a moderate impact of the spontaneous-braking behavior on the traffic congestion.

It can be seen that before the spontaneous-braking parameter was applied, the congestion already occurred on the traffic (Fig. 6). While in Fig. 7, the effect of spontaneousbraking on traffic congestion just a slightly impact is shown. That because in density value $\rho=0.75$, the traffic congestion already appeared although in the condition without spontaneous-braking behavior.

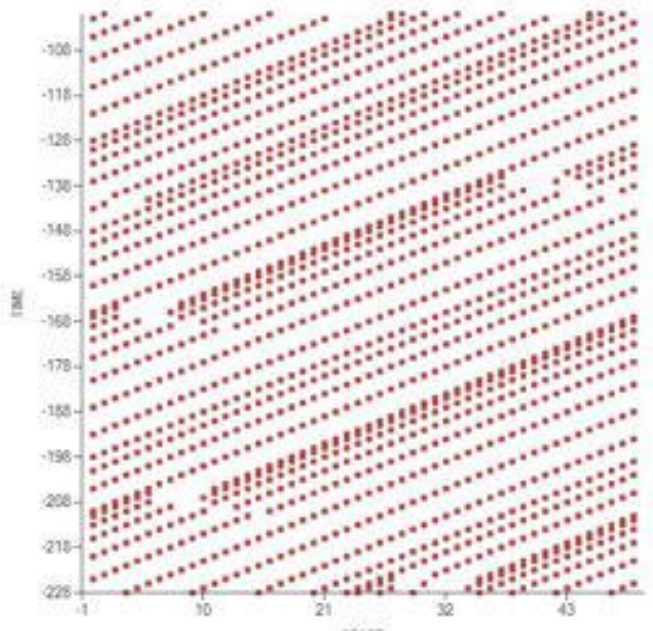

(a) yuce

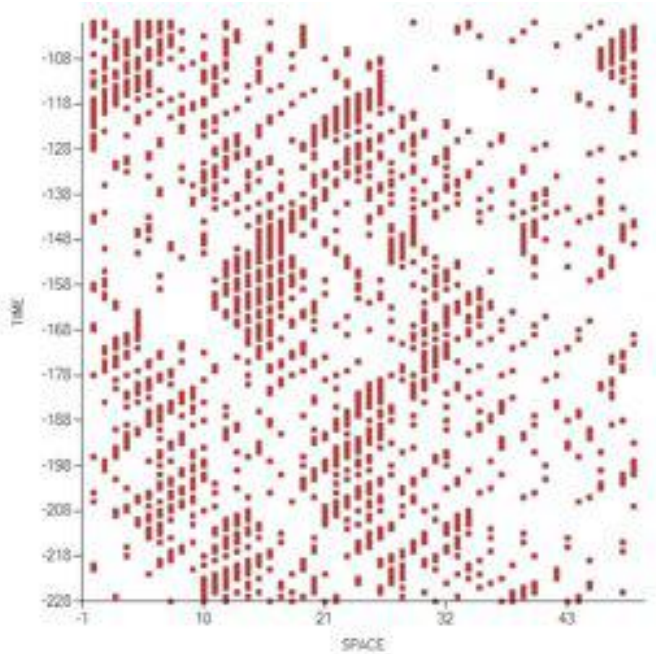

(b)

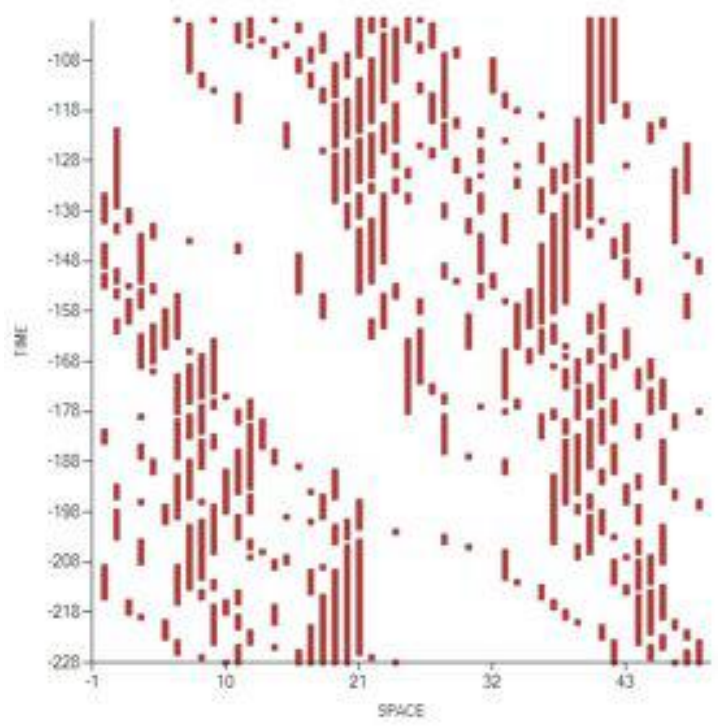

(c)

Figure 5. Space-time diagram for density $\rho=0.25$ and $\mathrm{P}_{\mathrm{b}}=0(\mathrm{a}), \mathrm{P}_{\mathrm{b}}=0.3$

(b), and $\mathrm{P}_{\mathrm{b}}=0.7$ (c); without lane-changing maneuvers 
(a)
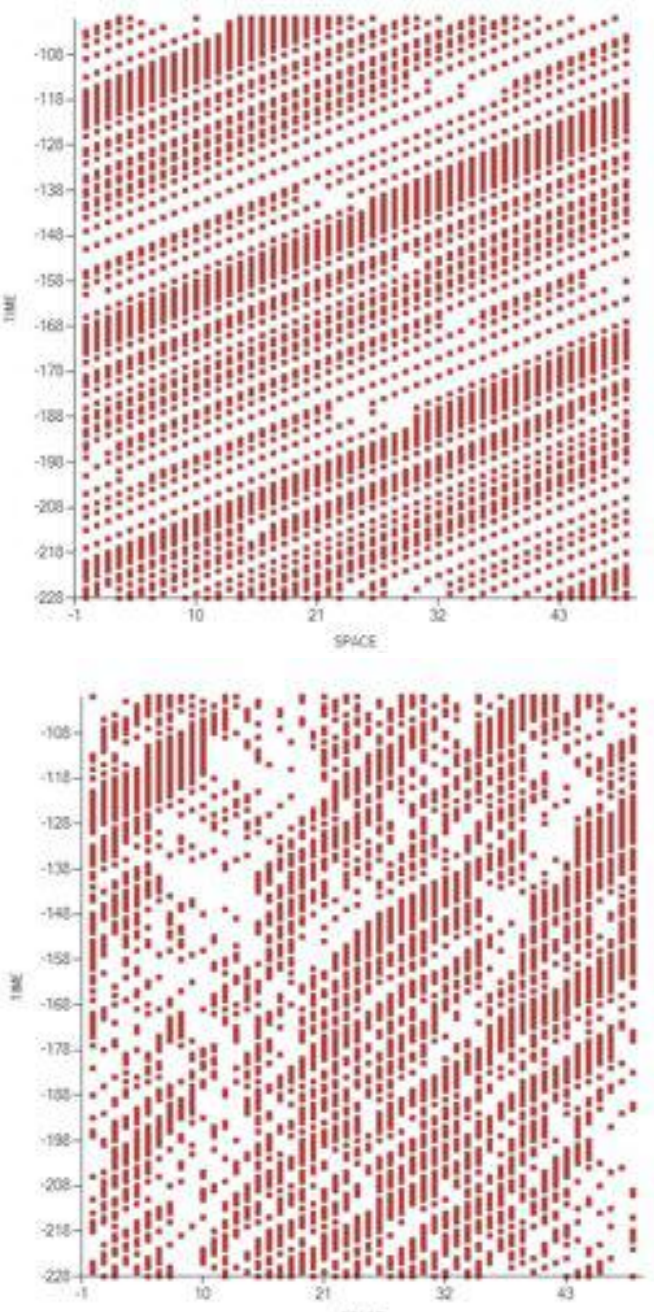

(b)

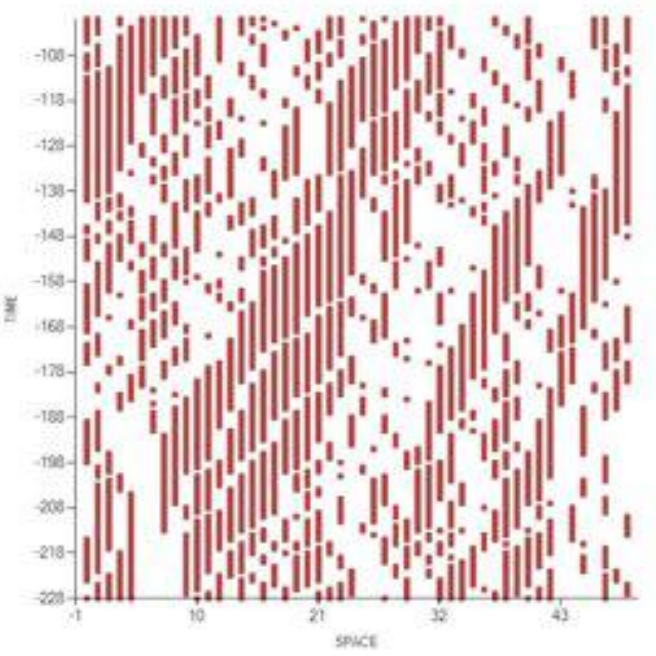

Figure 6. Space-time diagram for density $\rho=0.50$ and $P_{b}=0$ (a), $P_{b}=0.3$ (b), and $\mathrm{P}_{\mathrm{b}}=0.7$ (c); without lane-changing maneuvers

The lane-changing effect on traffic congestion is discussed from here. As shown before that the spontaneous-braking behavior can contribute to the traffic congestion.

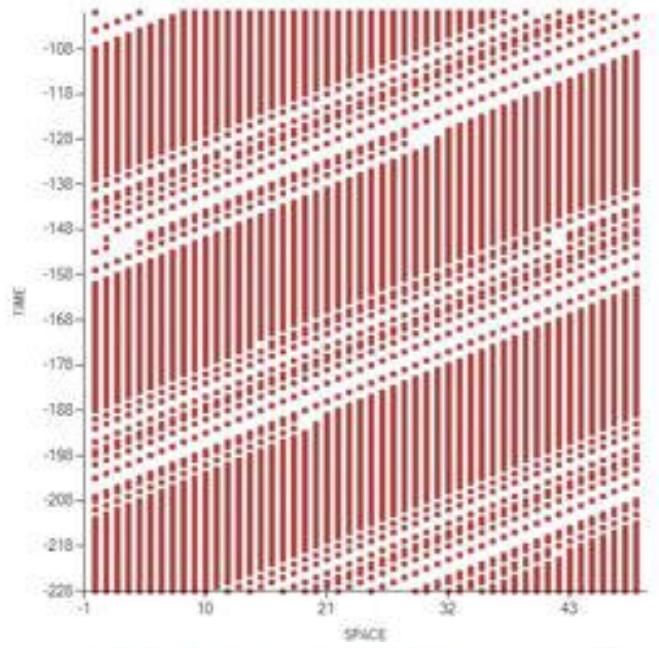

(a)

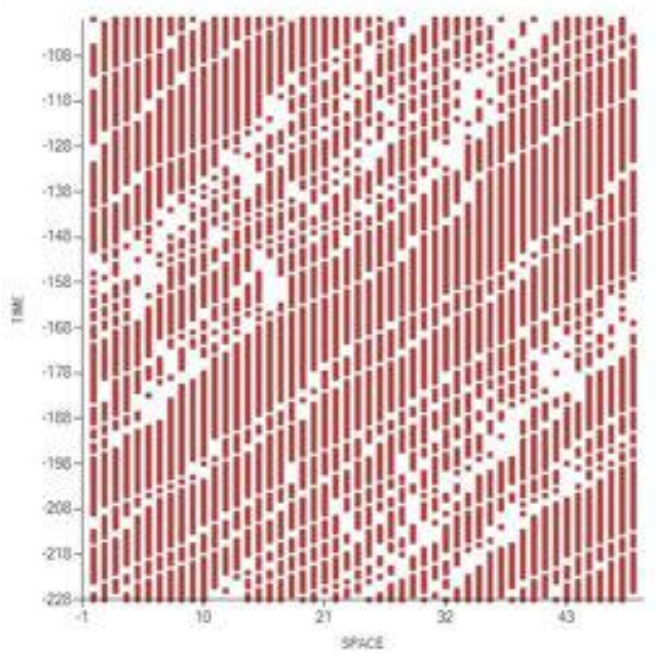

(b)

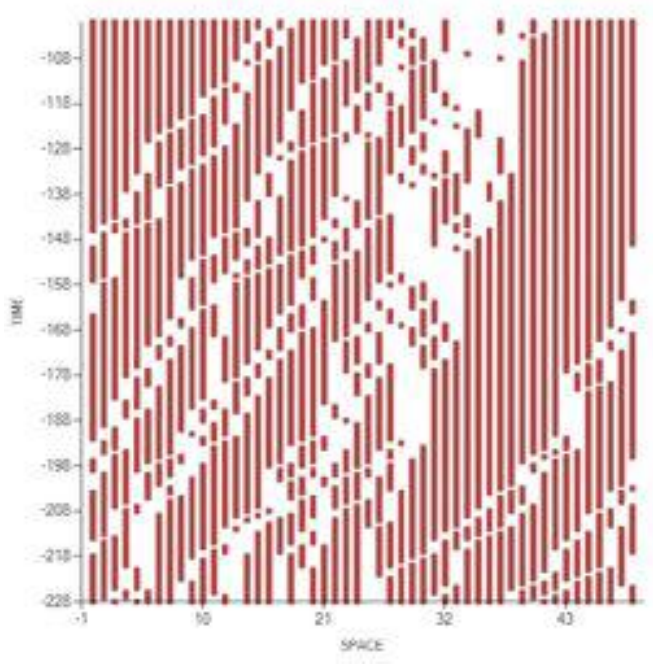

(c)

Figure 7. Space-time diagram for density $\rho=0.75$ and $P_{b}=0(a), P_{b}=0.3(b)$, and $\mathrm{P}_{\mathrm{b}}=0.7(\mathrm{c})$; without lane-changing maneuvers

Therefore, in this section we evaluate the effect of lanechanging to reduce the congestion level. This lane-changing model was applying the equations (8), (9), and (10). 
In this simulation, the vehicles can look back and estimate the situation along 5 cells behind on the other lane before make a lane-changing. We provide a set of space-time diagrams in Fig. 8, Fig. 9, and Fig. 10 for the density values $\rho$ $=0.25 ; 0.50$; and 0.75 .

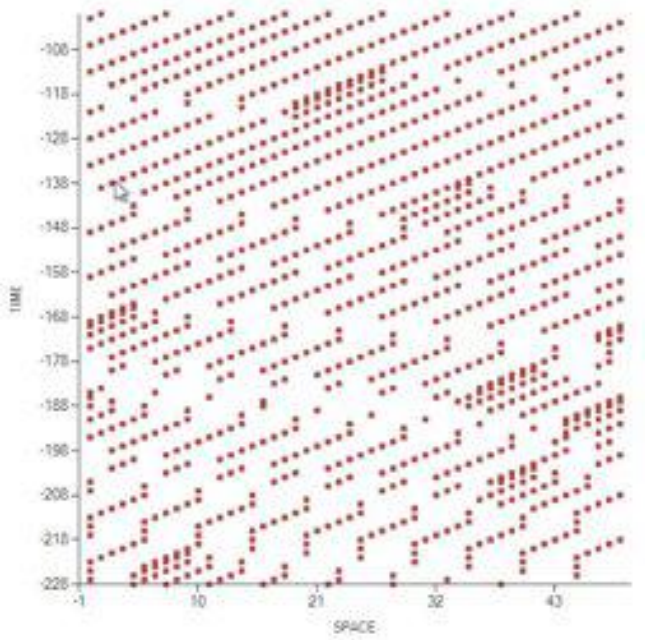

(a)

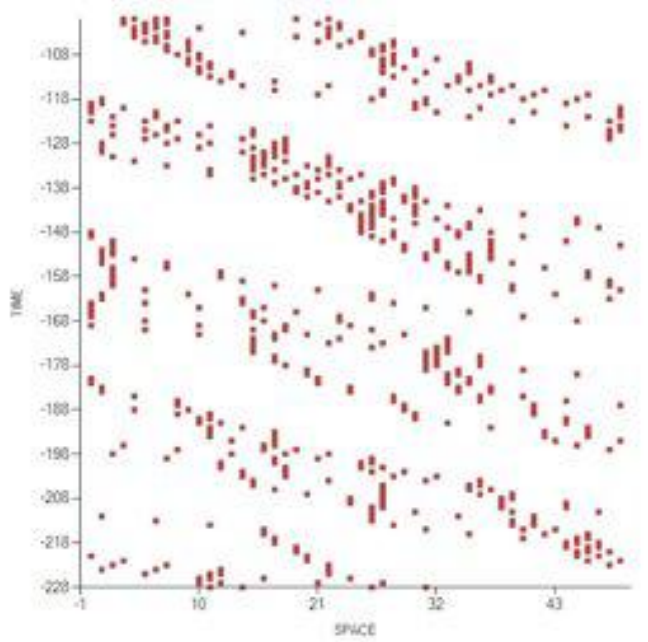

(b)

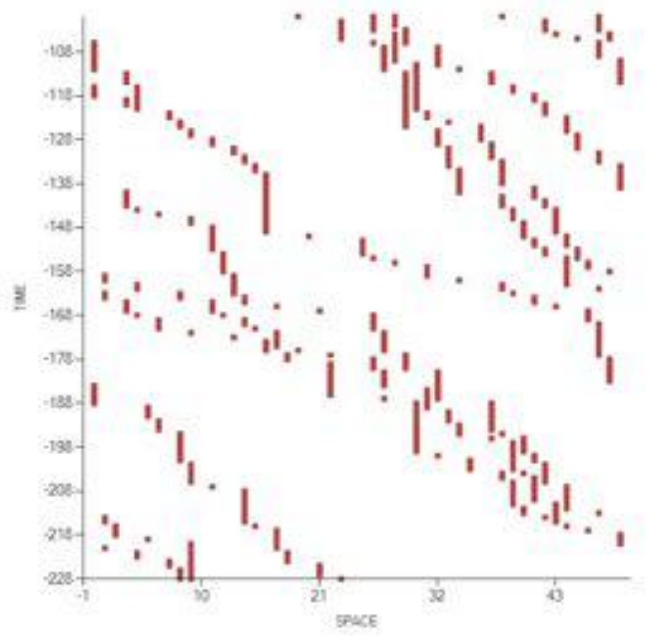

(c)

Figure 8. Space-time diagram for density $\rho=0.25$ and $\mathrm{P}_{\mathrm{b}}=0(\mathrm{a}), \mathrm{P}_{\mathrm{b}}=0.3(\mathrm{~b})$, and $\mathrm{P}_{\mathrm{b}}=0.7(\mathrm{c})$; with lane-changing maneuvers

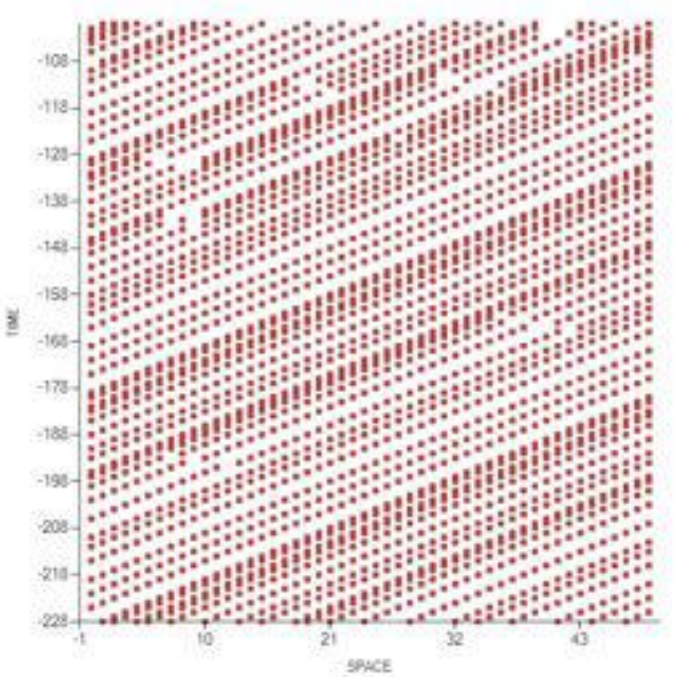

(a)

(b)

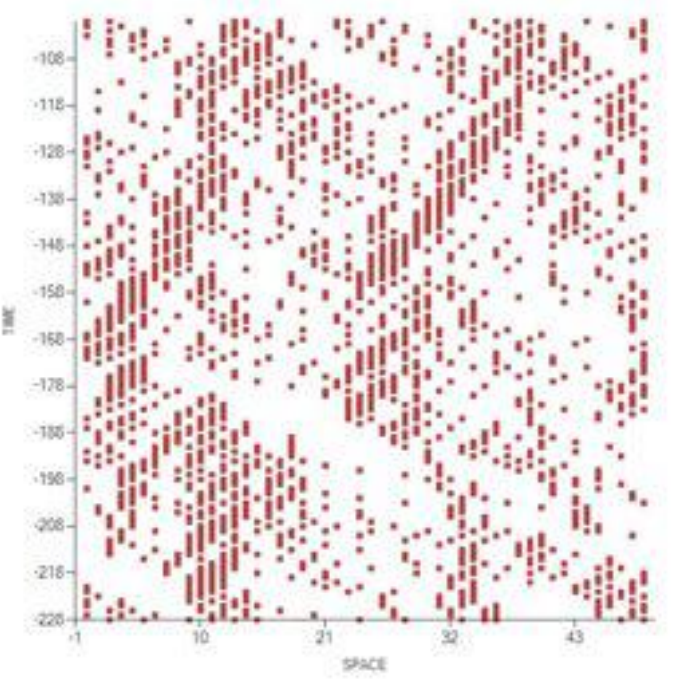

(c)

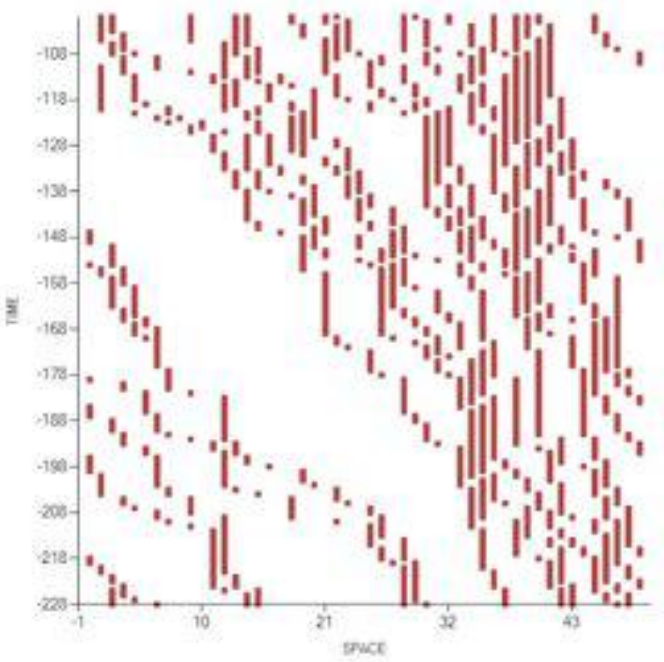

Figure 9. Space-time diagram for density $\rho=0.50$ and $\mathrm{P}_{\mathrm{b}}=0(\mathrm{a}), \mathrm{P}_{\mathrm{b}}=0.3(\mathrm{~b})$, and $\mathrm{P}_{\mathrm{b}}=0.7$ (c); with lane-changing maneuvers 


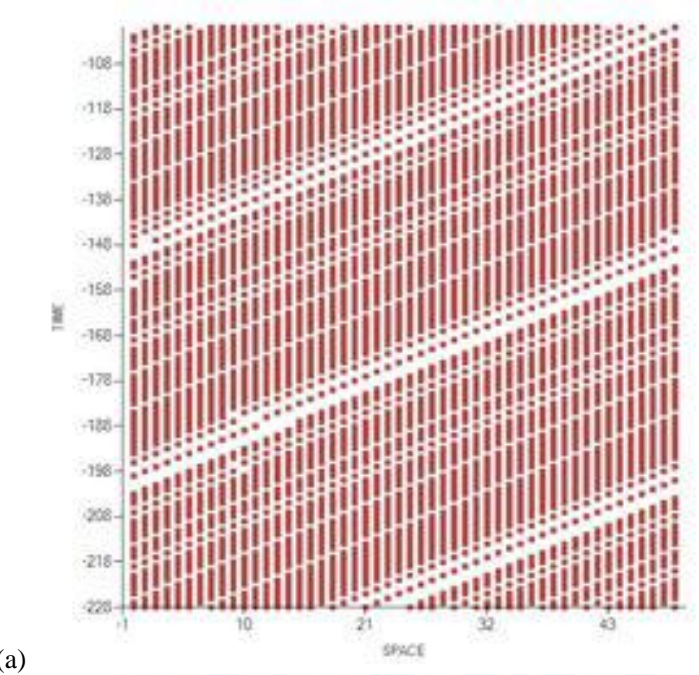

(a)

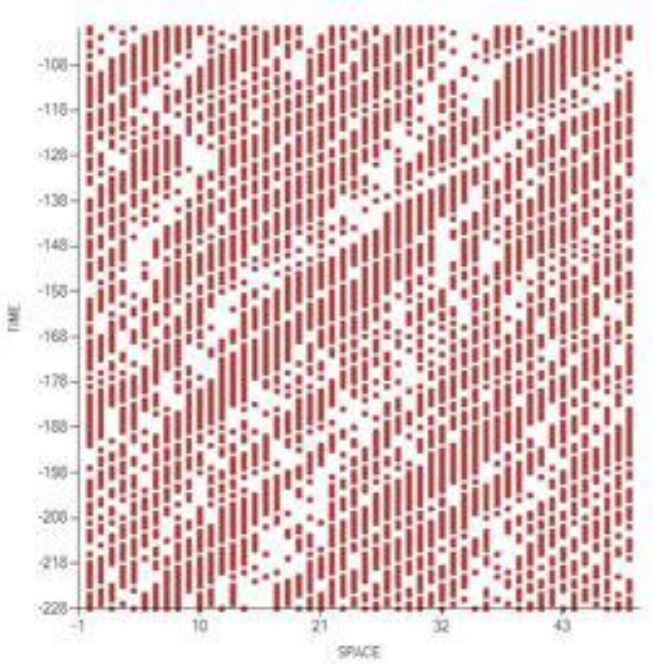

(b)

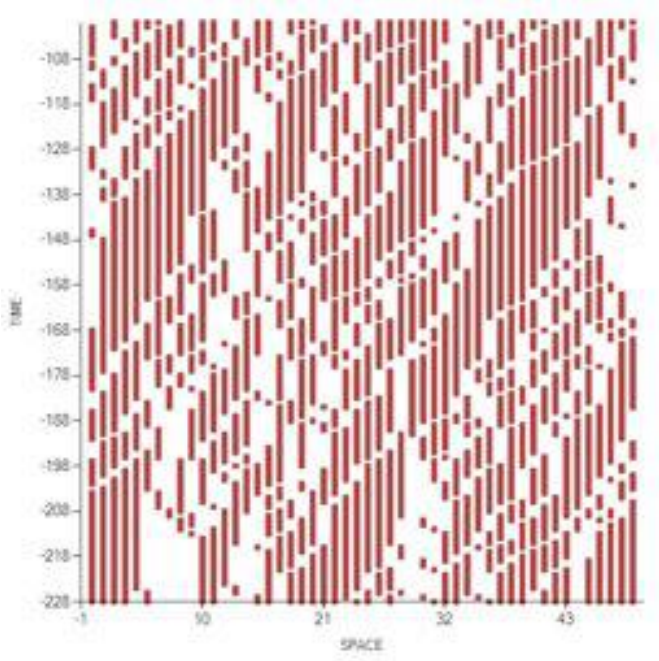

(c)

Figure 10. Space-time diagram for density $\rho=0.75$ and $P_{b}=0$ (a), $P_{b}=0.3$ (b), and $\mathrm{P}_{\mathrm{b}}=0.7$ (c); with lane-changing maneuvers.

The comparative graph shows that for the traffic density $\rho$ $<0.75$, the lane-changing maneuvers have given a good impact to reduce the congestion level. However, in all spontaneous-braking parameter value condition, the result shows that there is no significant impact that is contributed by lane-changing maneuver.

\section{CONCLUSION}

In this work, we simulate the braking behavior of the driver and present the new Cellular Automata model for describing this characteristic. The original NaSch model has been modified to accommodate the parameter of spontaneousbraking probability. This spontaneous-braking probability rule captures the natural of braking behavior due to human behavior. This simulation shows that the traffic congestion can be caused not only by the road capacity condition but also by driver behavior. Moreover, we also evaluate the effect of lanechanging to reduce the congestion that is caused by the parameter of spontaneous-braking probability.

\section{REFERENCES}

[1] K. Nagel and M. Schreckenberg, "A cellular automaton model for freeway traffic," Journal of Physics I France, vol. 2, no. 12, pp.22212229, 1992.

[2] A. Schadschneider and M. Schreckenberg. "Cellular automaton models and traffic flow," Physics A, 1993.

[3] L. Villar and A. de Souza, "Cellular automata models for general traffic conditions on a line," Physica A, 1994.

[4] M. E. Lárraga, J. a. D. Río, and L. Alvarez-lcaza, "Cellular automata for one-lane traffic flow modeling," Transportation Research Part C: Emerging Technologies, vol. 13, no. 1, pp. 63-74, Feb. 2005.

[5] K. Nagel, "Particle hopping models and traffic flow theory," Physical review. E, vol. 53, no. 5, pp. 4655-4672, May 1996.

[6] K. Arai and Tri Harsono Agent and diligent driver behavior on the carfollowing part of the micro traffic flow in a situation of vehicles evacuation from Sidoarjo Prong roadway, International Journal of Computer Science and Network Security, 11, 1, 137-144, 2011.

[7] K. Arai, Tri Harsono, Ahmad Basuki, "Car-Following Parameters by Means of Cellular Automata in the Case of Evacuation," International Journal of Computer Science and Security (IJCSS), Vol (5), 2011.

[8] M. Rickert, K. Nagel, M. Schreckenberg, and A. Latour, "Two Lane Traffic Simulations using Cellular Automata," vol. 4367, no. 95, 1995.

[9] W. Knospe, L. Santen, A. Schadschneider, and M. Schrekenberg, "Disorder effects in cellular automata for two lane traffic," Physica A, vol. 265, no. 3-4, pp. 614-633, 1998.

[10] A. Awazu, "Dynamics of two equivalent lanes traffic flow model: selforganization of the slow lane and fast lane," Journal of Physical Society of Japan, vol. 64, no. 4, pp. 1071- 1074, 1998.

[11] E. G. Campri and G. Levi, "A cellular automata model for highway traffic," The European Physica Journal B, vol. 17, no. 1, pp. 159-166, 2000.

[12] L. Wang, B. H. Wang, and B. Hu, "Cellular automaton traffic flow model between the Fukui-Ishibashi and Nagel- Schreckenberg models," Physical Review E, vol. 63, no. 5, Article ID 056117, 5 pages, 2001.

[13] B. Jia, R. Jiang, Q. S. Wu, and M. B. Hu, "Honk effect in the two-lane cellular automaton model for traffic flow," Physica A, vol. 348, pp. 544 $552,2005$.

[14] D. Chowdhury, L. Santen, and A. Schadschneider, "Statistical physics of vehicular traffic and some related systems," Physics Report, vol. 329, no. 4-6, pp. 199-329, 2000.

[15] W. Knospe, L. Santen, A. Schadschneider, and M. Schreckenberg, "A realistic two-lane traffic model for highway traffic," Journal of Physics A, vol. 35, no. 15 , pp. 3369-3388, 2002.

[16] D. Chowdhury, L. Santen, and A. Schadschneider, "Statistical physics of vehicular traffic and some related systems," Physics Report, vol. 329, no. 4-6, pp. 199-329, 2000.

[17] R. J.Harris and R. B. Stinchcombe, "Ideal and disordered two- lane traffic models," Physica A, vol. 354, no. 1-4, pp. 582-596, 2005. 
[18] X. G. Li, B. Jia, Z. Y. Gao, and R. Jiang, "A realistic two-lane cellular automata traffic model considering aggressive lane- changing behavior of fast vehicle," PhysicaA, vol. 367, pp. 479-486, 2006.

[19] W. Knospe, L. Santen, A. Schadschneider, and M. Schreckenberg, "Empirical test for cellular automaton models of traffic flow," Phys. Rev. E, vol. 70, 2004.

[20] S. Maerivoet and B. D. Moor, "Transportation Planning and Traffic Flow Models," 05-155, Katholieke Universiteit Leuven, Department of Electrical Engineering ESAT-SCD (SISTA), July 2005.

AUTHORS PROFILE

Kohei Arai, He received BS, MS and PhD degrees in 1972, 1974 and 1982, respectively. He was with The Institute for Industrial Science and Technology of the University of Tokyo from April 1974 to December 1978 and also was with National Space Development Agency of Japan from January, 1979 to March, 1990. During from 1985 to 1987, he was with Canada Centre for Remote Sensing as a Post Doctoral Fellow of National Science and Engineering Research Council of Canada. He moved to Saga University as a Professor in Department of Information Science on April 1990. He was a councilor for the Aeronautics and Space related to the Technology Committee of the Ministry of Science and Technology during from 1998 to 2000. He was a councilor of Saga University for 2002 and 2003. He also was an executive councilor for the Remote Sensing Society of Japan for 2003 to 2005. He is an Adjunct Professor of University of Arizona, USA since 1998. He also is Vice Chairman of the Commission A of ICSU/COSPAR since 2008. He wrote 30 books and published 307 journal papers 